\title{
ESTRATEGIAS DE RECUPERACIÓN DE PREDIOS BALDÍOS EN LA CIUDAD DE TOLUCA. PROSPECTIVA DE ORDENAMIENTO URBANO
}

\section{STRATEGIES FOR THE RECOVERY OF VACANT LAND IN THE CITY OF TOLUCA. PROSPECTIVE OF URBAN PLANNING}

\section{Carlos David Sánchez Muñoz ${ }^{1}$}

\section{RESUMEN}

Este estudio propone estrategias que dan sustento normativo a la recuperación de los predios baldíos en la ciudad de Toluca, que carece de una política urbana adecuada, así como de un sustento legal acorde a las necesidades de la población. Los resultados muestran que el marco regulatorio de los usos de suelo urbano, no contempla estrategias de reinserción de los vacíos urbanos a la economía de la ciudad. Lo que agudiza su abandono por largos períodos de tiempo, la especulación comercial, la enajenación fraudulenta, la generación de focos de infección y la subutilización al amparo de la debilidad de la política urbana. Es imperante establecer mecanismos que coadyuven en la gestión del intercambio de plusvalías y reconstitución del espacio público en beneficio de la colectividad, a través de la participación social de forma directa, vinculada a la aplicación de políticas urbanas y la adhesión del marco normativo vigente. El procedimiento realizado en este estudio puede mejorarse y replicarse en otros municipios e inclusive a nivel nacional. Aplicamos el método analítico en la revisión de literatura, ordenamientos, planes, programas y la legislación vigente. EI método inductivo mediante recorridos de campo y una ficha técnica para actualizar la información de los baldíos urbanos.

Palabras clave: Ordenamiento Urbano, baldíos, estrategias, normatividad, derecho.

\section{ABSTRACT}

This study proposes strategies that give normative support to the recovery of vacant lots in the city of Toluca, which lacks an adequate urban policy, as well as legal support according to the needs of the population. The results show that the regulatory framework of urban land uses does not include

\footnotetext{
${ }^{1}$ Doctorante en Urbanismo Maestro en Derecho con Mención de Honor Grado en Derecho con Mención de Honor profesor en la cátedra de Derecho Ambietal en el Instituto Tecnológico de Tenango el profesor de la asignatura de Derecho Laboral y Civil en el Centro de Estudios Jurídicos del Estado de México Profesor de la asignatura de Derecho Fiscal y Derecho Mercantil en la Escuela Bancaria y Comercial. Afiliação: Universidad Autónoma del Estado de México. ORCID: https://orcid.org/0000-0001-8775-0135. E-mail: cd.sanchez002@ gmail.com.
} 
strategies for the reintegration of urban gaps into the city's economy. What exacerbates its abandonment for long periods of time, commercial speculation, fraudulent alienation, the generation of pockets of infection and underutilization under the weakness of urban policy. It is imperative to establish mechanisms that contribute to the management of the exchange of capital gains and reconstitution of public space for the benefit of the community, through social participation in a direct manner, linked to the application of urban policies and adherence to the current regulatory framework. The procedure performed in this study can be improved and replicated in other municipalities and even nationwide. We apply the analytical method in the literature review, regulations, plans, programs and current legislation. The inductive method through field trips and a technical sheet to update the information of urban wastelands.

Keywords: Urban planning, vacant, strategies, regulations.

\section{INTRODUCCIÓN}

En México la expansión progresiva de las ciudades y los desequilibrios en los componentes de los sistemas urbanos y metropolitanos, les dota de prioridad alta en las leyes federales, estatales y reglamentos municipales en materia de desarrollo urbano.

La Nueva Ley General de Asentamientos Humanos, Ordenamiento territorial y Desarrollo Urbano. Dispone ordenar la expansión urbana a través de la coordinación de los tres niveles de gobierno y las facultades de los municipios y los principios para determinar las provisiones, reservas, usos del suelo y destinos de áreas y predios que regulan la propiedad, creación de instrumentos que garanticen la corresponsabilidad del gobierno y la ciudadanía en la formulación, seguimiento y evaluación de la política pública en la materia (EUM-PR, 2016:1). La función pública confluye en tres instrumentos de intervención, la política de ordenamiento territorial, tiene por objeto equilibrar el desarrollo socioeconómico y desarrollo urbano; mejoramiento de la calidad de vida de la población, gestión responsable de los recursos naturales y protección del medio ambiente (Contreras Gatica, 2011). A través de las políticas los gobiernos lideran las redes de actores que participan en los problemas y toman decisiones para la consecución de objetivos (Jessop, 2002), (Blanco I. , 2009). Uno los objetivos es gestionar la competitividad urbana, a través de la coordinación de actores públicos y privados, inversión eficiente en obra pública y captación de inversiones privadas (Ana, 2004). El programa nacional de ordenamiento territorial y desarrollo Urbano conjunta actividades, servicios y procesos de regulación que llevan a la materialización de la política de ordenamiento territorial. El 
proyecto (s) unidad específica de intervención, agrupa las actividades generadoras de bienes y servicios tendentes a la satisfacción de necesidades sociales (Nieves, 2006).

En el contexto del sistema global urbano, algunos gobiernos latinoamericanos implementan la política de intercambio de plusvalías a través de mecanismos fiscales para regular los usos de suelo, los permisos de urbanización e incrementos en el valor de la tierra. Aplican tasas impositivas diferenciadas para incentivar la mezcla de usos del suelo o imponen diversos tipos de exacciones o cargas. La política de intercambio de plusvalías integra instrumentos regulatorios y fiscales. El cuerpo normativo o regulatorio conlleva un impuesto o contribución en especie, el que paga el propietario a cargo del incremento en el valor del suelo. Los instrumentos fiscales (impuestos y contribuciones), gravan a los propietarios de los predios (Smolka \& Amborski, Recuperación de plusvalías para el desarrollo urbano: una comparación inter- americana, 2003). En el ámbito nacional la recuperación de baldíos a través de la política de intercambio de plusvalías, es reciente. En el año 2000 se implementó en los estados de Aguascalientes, Coahuila, Michoacán, Morelos, Nuevo León, San Luis Potosí y Sinaloa (Fernández, 2006), recientemente en los estados de Yucatán y Sonora. Los casos indican que el intercambio de plusvalías se aplica como estrategia de control de predios baldíos y responde a problemáticas variadas. En la ciudad de Mérida los baldíos se visualizan en un problema de sanidad. Llevando a la modificación del reglamento de terrenos baldíos e incremento de sanciones económicas por incumplimiento de obligaciones de mantenimiento de los terrenos, la multa se fijó de acuerdo a la zona y la superficie del predio, esta se elevaría de 10 a 100 y 200 salarios mínimos. Los beneficios proyectan eliminación de focos de infección e inseguridad, mejoramiento de la imagen urbana y calidad de vida de los ciudadanos. Las iniciativas abrieron la posibilidad de que el ayuntamiento otorgue en uso, comodato o renta los predios baldíos para aprovecharlos. Aunque se propuso eliminar la expropiación de predios privados, la atribución es de orden federal, el ayuntamiento no tiene facultades (Castillo, 2015). En Sonora la ausencia de control sobre los predios baldíos, estos representan $38 \%$ del total de propiedades consignadas en el registro público de la propiedad. Motivo que se legislará el uso y disposición de este tipo de inmuebles. La prospectiva determino un proyecto específico sobre inventario y contabilidad de los inmuebles de la federación, estados y municipios. El alcance de los objetivos acota optimizar el aprovechamiento del suelo baldío y promover proyectos de impacto urbano, generadores de inversión y empleos. Para ello se realizó investigación legal, técnica y administrativa; verificación de campo, identificación geográfica y valuación comercial (Sonora, 2015).

En el caso del Estado de México y municipios, las prioridades van de la mano del marco federal en materia de ordenamiento territorial y urbano. A los municipios se les otorgan facultades para la formulación, aprobación, ejecución, evaluación y modificación de los planes municipales de 
desarrollo urbano; determinación de uso y aprovechamiento del suelo, acciones de conservación, mejoramiento y crecimiento de los centros de población.

En el caso de Toluca, el plan municipal de desarrollo urbano establece un programa de fomento a la ocupación de los baldíos urbanos, mediante el predial base suelo y asistencia jurídica para la regularización (GEM-PDMT, 2013: 325-326). Sin embargo la iniciativa enunciativa, carece de fundamento analítico y legal para la operación. La recuperación de los predios baldíos en la ciudad de Toluca se presenta como oportunidad para promover la densificación urbana, optimización de la infraestructura y equipamiento, mejoramiento de la imagen urbana e ingresos municipales por servicios y equipamiento. El suelo urbano vacante podría reinsertarse en la dinámica de la ciudad mediante la gestión y políticas fiscales que desaliente la retención del suelo por parte de los propietarios con capacidad económica (Godoy, 2006), amorticen las inversiones públicas e impuestos de los contribuyentes destinados a la infraestructura y equipamiento urbano.

\section{MÉTODOS Y MATERIALES}

Los predios baldíos o vacantes refieren los terrenos remanentes en el ámbito urbano y reservas territoriales periféricas a las ciudades. En este estudio el objeto de análisis es el baldío (s) urbano (s), aquel que se localiza en los intersticios de la ciudad de Toluca, carecen de edificación y valor de uso cierto; cuya recuperación se vincula a la función pública de ordenamiento urbano.

\section{Fase analítica}

La recuperación de los predios baldíos coloca en el centro el uso actual del suelo; en el extremo derecho el marco legal, en el extremo izquierdo la rentabilidad y en la cúspide el acondicionamiento urbano de la localización.

El uso del suelo engloba los aspectos que repercuten en la funcionalidad futura de los terrenos: localización, potencialidades y restricciones de uso precedente, planeamiento urbanístico y comercialización (UBA, 2000). El marco legal sustenta la certidumbre jurídica de la propiedad, la rentabilidad comprende el valor del suelo y capitalización a futuro. El acondicionamiento urbano se integra por el equipamiento y los servicios disponibles de la localización de los terrenos. El modelo interpretativo de la investigación, identifica los factores que inciden en la recuperación de los predios baldíos en la ciudad de Toluca (Figura 1). 
Figura 1. Modelo interpretativo.

VARIABLES INDEPENDIENTES

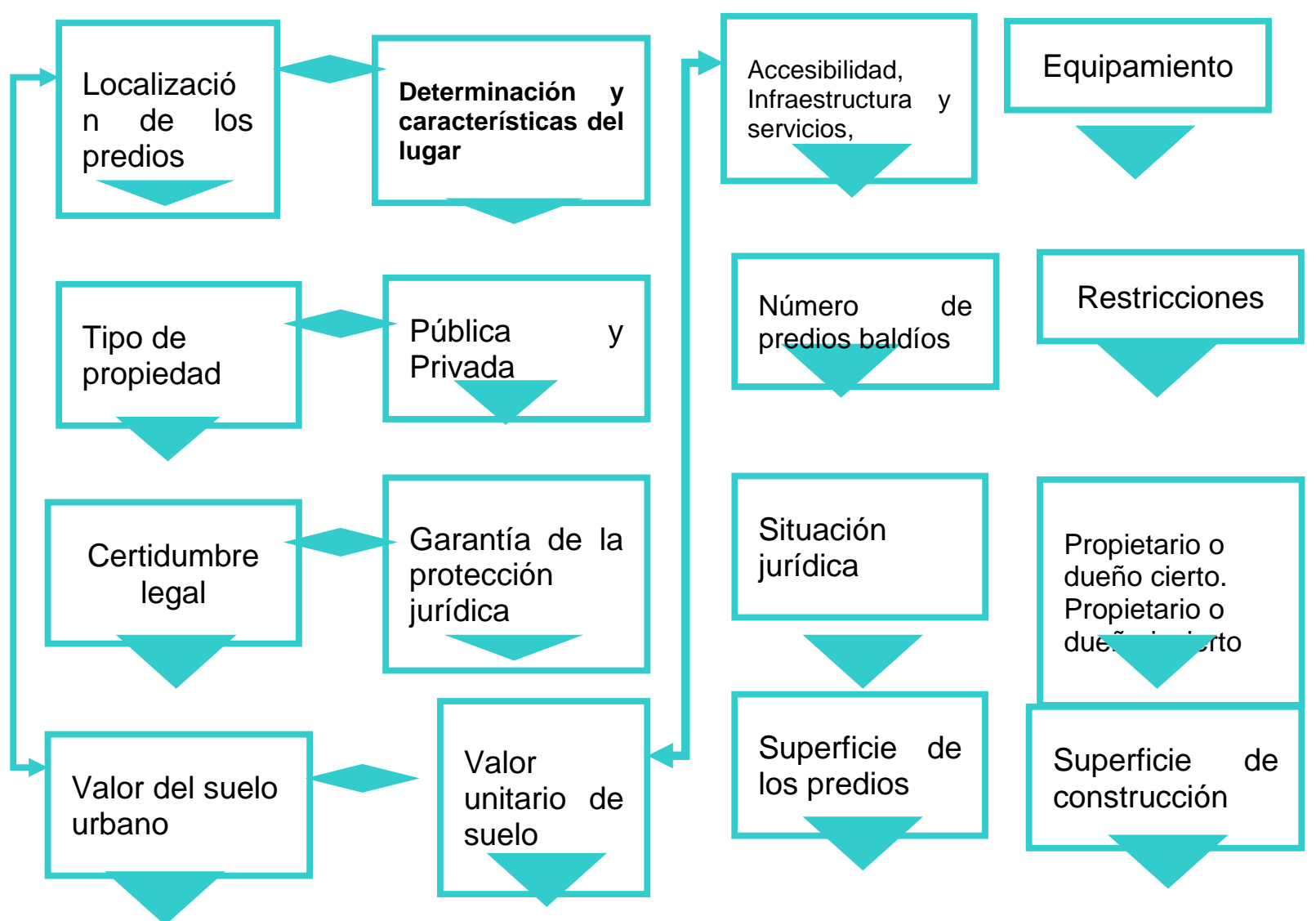

Elaboración propia

Localización geográfica cumple una doble función, será limitante, si los predios se ubican en zonas de riesgo para la salud e integridad física de la población -riesgos físicos, químicos y sanitarios- La influencia positiva o impulso depende de la accesibilidad y la calidad de la infraestructura y equipamiento urbano. La propiedad identifica los bienes inmuebles de dominio público y los de dominio privado. La propiedad privada es potestad del individuo, amparada en la estructura de derecho, la desocupación de los inmuebles privados se atribuye a la especulación económica. La desocupación de los bienes públicos es función del traslado de actividades estatales de un lugar a otro; cierre de edificaciones obsoletas o edificaciones históricas degradadas. Los monumentos arqueológicos, artísticos e históricos están protegidos para conservarlos, restaurarlos y recuperarlos. Las declaratorias se publican en el Diario Oficial de la Federación o periódicos oficiales de entidades federativas. El Presidente de la República a través de la Secretaría de Educación Pública, expide o revoca la declaratoria. Los propietarios de inmuebles históricos cuando resuelvan acondicionarlos 
deberán solicitar autorización y apegarse a los requisitos de seguridad y control que fija el reglamento correspondiente. La certidumbre legal es la certeza jurídica de la propiedad que se posee íntegramente. Las limitantes identifican la incertidumbre en la tenencia de los inmuebles. En los predios intestados, los posibles herederos dispondrán del bien, siempre que se concluya el juicio sucesorio. En casos que se carece de propietario cierto, no se tiene inscripción en el registro público, por tanto, el bien no pertenece a nadie. El valor de suelo expone que la expectativa de elevar ganancias por la venta de los inmuebles, ocasiona que los propietarios mantengan los predios desocupados por largos períodos de tiempo. Si no se cubren las expectativas, no se estará en disposición para vender el inmueble. El valor económico se elevará en razón de la accesibilidad al centro y equipamiento urbano.

\section{FASE EMPÍRICA}

En el estudio se utilizó un listado de terrenos baldíos (Sánchez, 2011), el cual se verificó y actualizó en los recorridos de campo. Se aplicó una ficha técnica en el año 2014, para registrar la problemática y características de los predios. La propiedad y la superficie se determino con datos del Instituto de Geografía, Estadística y Catastro del Estado de México.

\section{Caso de estudio}

El municipio de Toluca colinda al norte con Almoloya de Juárez, Temoaya y Otzolotepec; al oriente Lerma, San Mateo Atenco y Metepec; al sur Metepec, Calimaya, Tenango del Valle, poniente Zinacantepec y Almoloya de Juárez (México, Plan de Desarrollo Urbano del Municipio de Toluca, 2003), (Figura 2).

Figura 2. Localización del municipio Toluca.

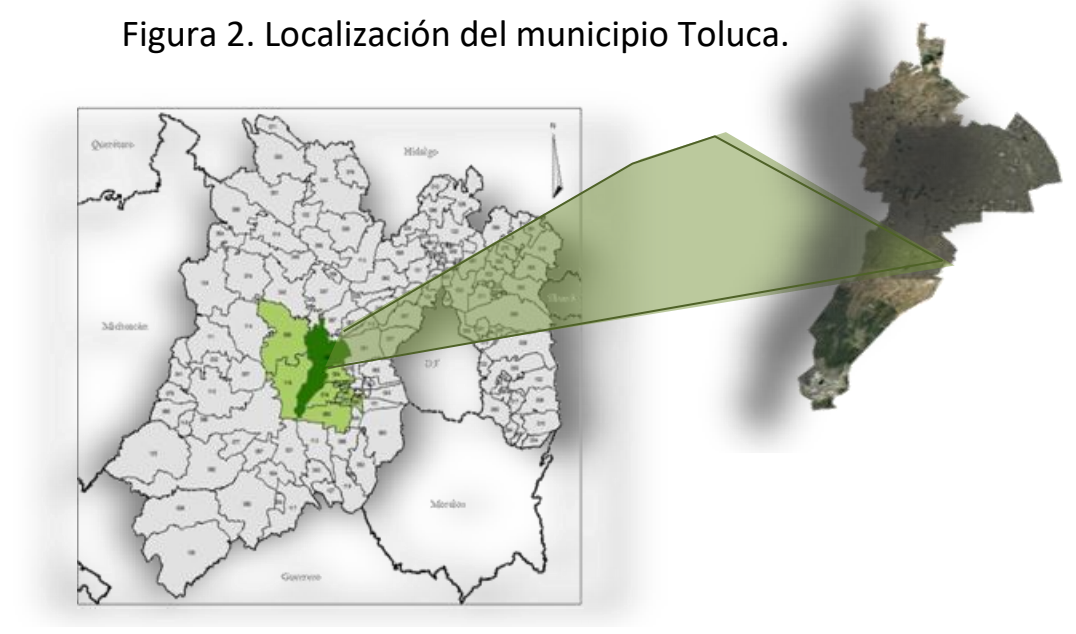

Fuente: Coespo, 2010 
El municipio tiene una extensión de 456.17 kilómetros cuadrados ${ }^{2}$, representa $2.0 \%$ del territorio estatal. Se Integra por 100 localidades, 819 mil 561 habitantes y 1,796.61 habitantes por kilómetro ${ }^{2}$. 94.0\% de la población reside en localidades urbanas. La inmigración de pobladores de la Ciudad de México, en las últimas tres décadas ha incrementado la demanda de suelo habitacional.

La ciudad de Toluca de Lerdo es centro político- administrativo de los gobiernos municipal y estatal. Núcleo urbano, comercial y de servicios más importante de la quinta zona metropolitana del país. Cuenta con 489,333 habitantes, 52.4\% mujeres, $47.6 \%$ hombres, 124,951 viviendas habitadas, dispone de 4,713 manzanas y 2,151 vialidades, $18 \%$ de las vialidades no tiene recubrimiento, en la zona metropolitana el déficit se eleva a 44\% (Instituto Nacional de Geografía Estadística e Info, 2010), (INEGI, 2014) El área urbana se integra por el centro histórico, ciento seis colonias y barrios antiguos: Santa Clara, la Merced (Alameda), San Sebastián, San Bernardino, La Retama, San Miguel Apinahuizco, Teresona, Zopilocalco, el Coporo, Santa Bárbara, Huitzila, San Luis Obispo, Tlacopa y San Juan Bautista (Orozco Hernández \& Tapia Quevedo). El área de observación delimita el polígono conformado por El Paseo Matlatzincas o Paseo Tollocan norte, Paseo Tollocan sur, al occidente el vértice formado por Paseo Tollocan, Adolfo López Mateos y Paseo Matlatzincas y al oriente Paseo Tollocan (Figura 3).

\section{Figura 3. Delimitación del área de estudio}

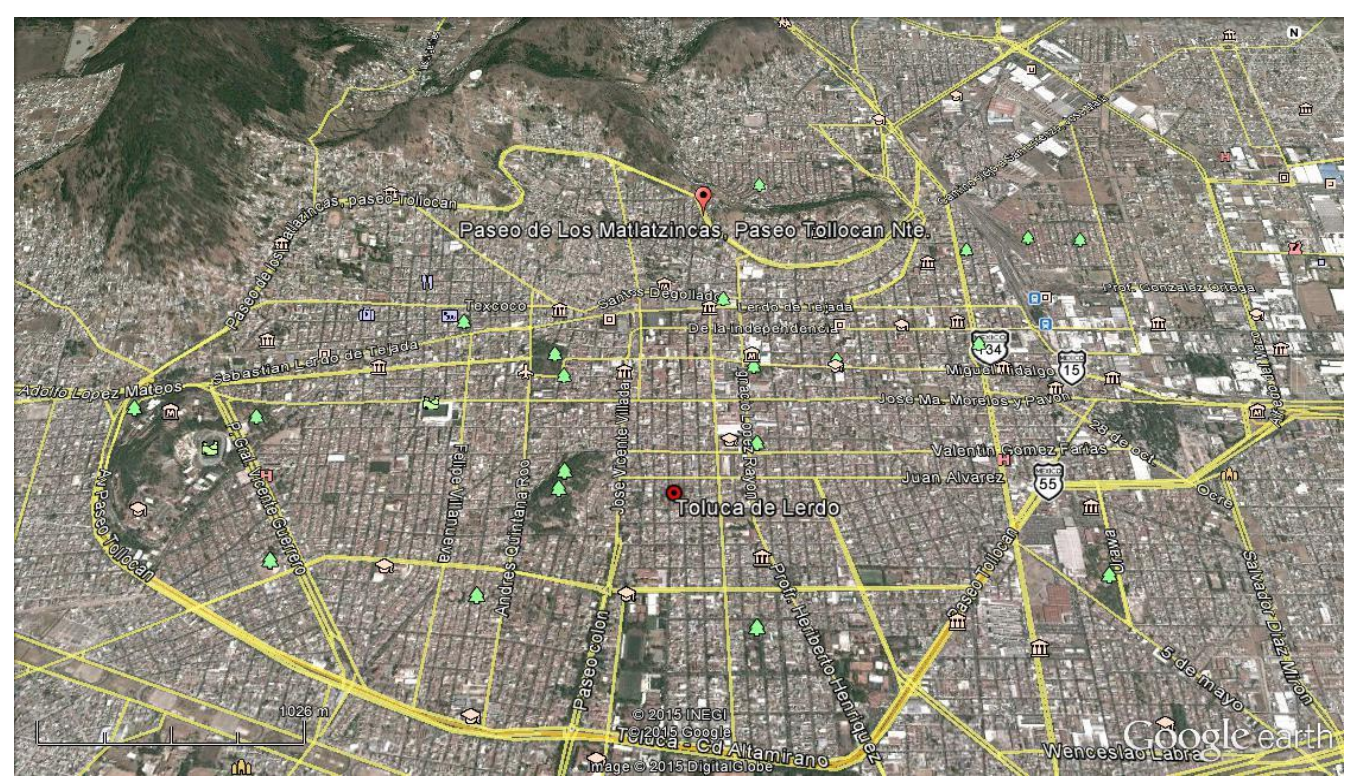

Fuente: (Earth, 2015). 


\section{VERIFICACIÓN DE PREDIOS BALDÍOS}

A partir del listado de Sánchez et al (2011) se identificaron y verificaron 180 baldíos. En propiedad del ayuntamiento $2 \%$; $96 \%$ propiedad privada y propiedad indefinida $2 \%$. La superficie total cubre $160,717.31 \mathrm{~m}^{2}$ o 16.07 has.

La propiedad pública identifica el baldío 53, ubicado sobre la bóveda del río Verdiguel, el cual se desalojo y recuperó por alta probabilidad de hundimiento (Cuadro 1).

Cuadro 1. Baldíos Ayuntamiento de Toluca

No. Registro

53

68

102

160

4
Ubicación

José Ma. Morelos esq. Gral. Agustín

Millán

Tianguistenco casi esq. Tenancingo

Lerdo de Tejada esq. 21 de Marzo

Paseo Tollocan entre Fidel

Velásquez y Sotero Prieto
Superficie $\mathrm{m} 2$

369.67

168.07

364.59

$1,098.25$

$2,000.58$

\section{TRABAJO DE CAMPO}

Los predios 68 y 160 cuentan con barda y el predio 102 tiene fachada histórica. El universo de predios privados se agruparon en cinco rangos de superficie: mayor a 5,000 $\mathrm{m}^{2} ; 2,000-5,000 \mathrm{~m}^{2}$; $1,000-2,000 \mathrm{~m}^{2} ; 500-1000 \mathrm{~m}^{2} ; 100-500 \mathrm{~m}^{2}$.

Figura 4. Grupos de predios baldíos privados

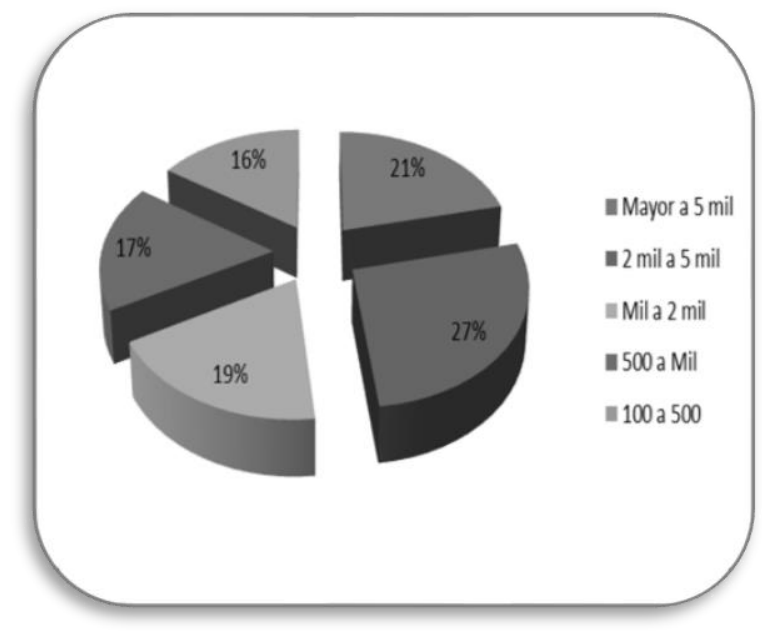

Elaboración propia 
El primero agrupa cuatro predios que conjuntan $31,189.72$ metros $^{2}$. El predio 77 identifica la antigua fábrica de jabón, considerada en un proyecto de rescate histórico e implantación de nuevos usos y funciones comerciales.

Los demás predios no tienen barda perimetral o bien se utilizan temporalmente, el predio 88 se habilita como campo de futbol (Cuadro 2).

Cuadro 2. Baldíos con superficie mayor a cinco mil $\mathrm{m}^{2}$

$\begin{array}{ccc}\begin{array}{c}\text { No. De Predio } \\ 77\end{array} & \text { Localización } & \text { Superficie } \\ & \text { Hidalgo y Lerdo de Tejada casi esq. } & 7,827.10 \\ 86 & \text { Tenango } & \\ 88 & \text { Paseo Matlazincas y Agustín Millán } & 8,439 \\ & \text { Lerma, Cuautitlán y Paseo de los } & 6,454.88 \\ 110 & \text { Matlazincas } & \\ & \text { Ilhuicamina y Eduardo Mendieta } & 8,468.74\end{array}$

Trabajo de campo

El segundo rango agrupa doce baldíos y superficie de $39,136.25 \mathrm{~m}^{2}$, el $60 \%$ no tiene barda, $25 \%$ bardado y el resto barda inconclusa. El 50\% de los predios se ubica en la Colonia Sánchez, la cual se originó por expansión del primer cuadro de la ciudad de Toluca. La ubicación de los baldíos con respecto al centro, les dota de ventajas comparativas; pero la pendiente dificultad la urbanización.

El tercero comprende 20 predios y superficie de $27,323.71 \mathrm{~m}^{2}$. El $45 \%$ de se localiza en las inmediaciones de Paseo Matlatzincas o Paseo Tollocan Norte y colonia Sánchez, la mayoría no tiene barda, comparte la pendiente pronunciada, riesgo de deslaves y altos costos de urbanización. El 55\% de los predios tiene barda perimetral y se localizan en vialidades del primer cuadro de la ciudad de Toluca: Gómez Farías, Lerdo de Tejada, Miguel Hidalgo, Santos Degollado, Leona Vicario, López Rayón e Isabela Católica. Aunque las propiedades no están en venta explicita, el precio del suelo en esta zona es alto, los propietarios que pretenden vender se apoyan en la gestión de empresas inmobiliarias para concretar las transacciones. El cuarto rango se conforma por 38 baldíos y superficie de $25,355.95 \mathrm{~m}^{2}$. El $70 \%$ tiene barda perimetral; $26 \%$ se localiza en la contigüidad de la Colonia Sánchez y primer cuadro de la ciudad de Toluca. La mayoría (74\%) se localiza del centro de la ciudad y el círculo delimitado por El Paseo Tollocan en dirección sur, oriente y occidente. El quinto rango se conforma por 82 predios y por tres en los que no se identificó la propiedad. Ocupan una superficie de $22,742.75 \mathrm{~m}^{2}$, poco más de $70 \%$ cuenta con barda perimetral; $18 \%$ se ubica en la contigüidad de la Colonia Sánchez y primer cuadro de la ciudad (64\%), el resto en el perímetro de Paseo Tollocan. 


\section{ESTADO DE LOS PREDIOS BALDÍOS}

Las condiciones de los predios destacan que la mayoría se encuentran abandonados, con maleza y escombros. Seguidos por los que se han habilitado como estacionamientos y tiraderos de basura (Figura 5)

Figura 5. Estado de los predios baldíos

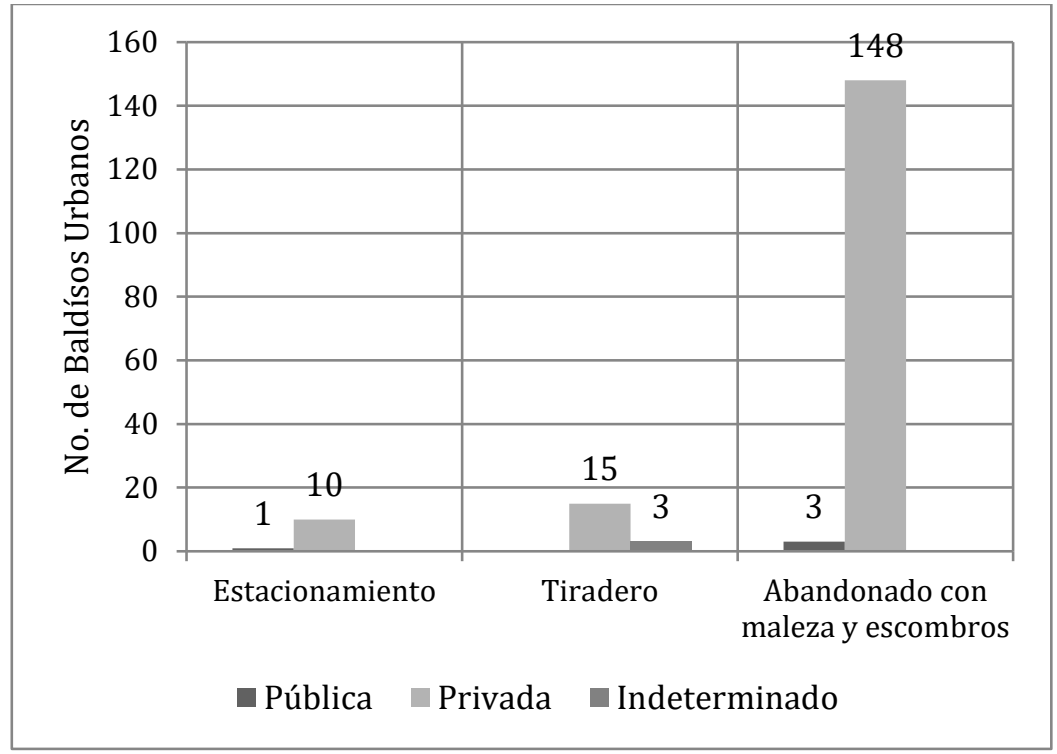

Elaboración propia con base en el trabajo de campo

Ante ausencia de barda perimetral, los baldíos se convierten en objetos de libre acceso para depositó de basura, focos de infección y fauna nociva, e inseguridad pública (Imagen 1).

Imagen 1. Baldíos sin barda

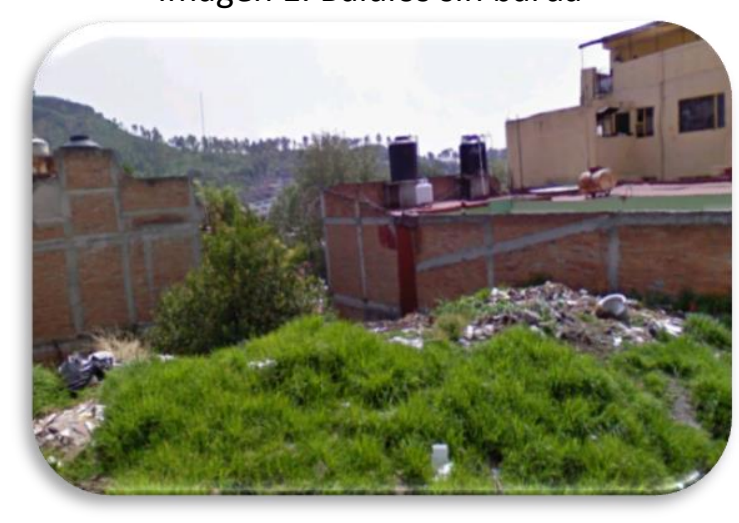

Imagen 2. Aprovechamiento como estacionamiento.

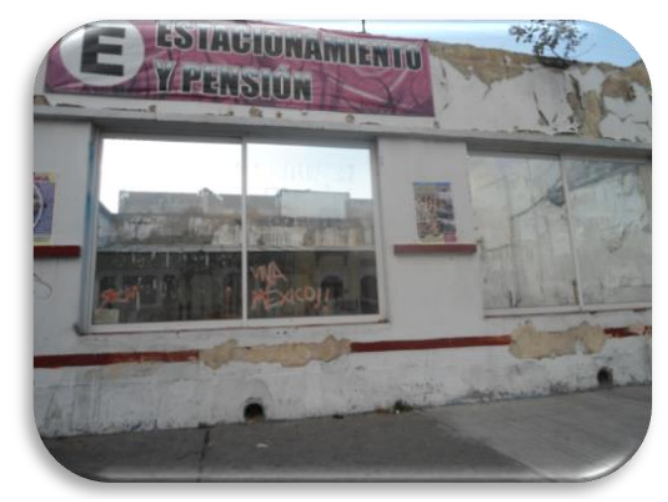


Imagen 3. Baldío con fachada histórica

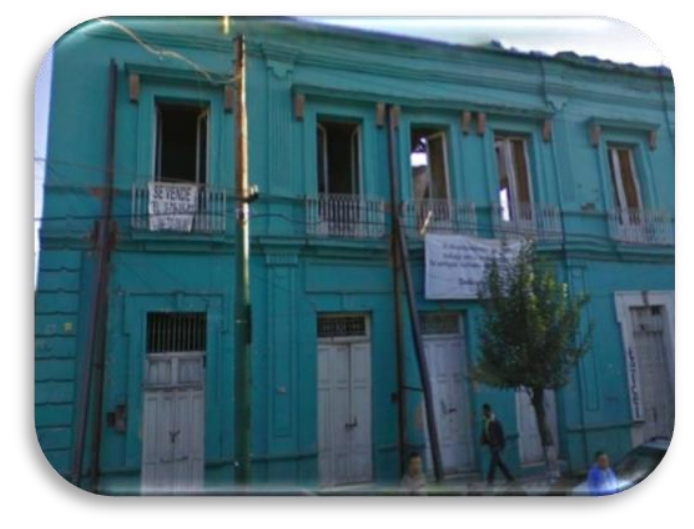

Los estacionamiento habilitados predios baldíos (Imagen 2), representan un negocio lucrativo, con baja inversión y altas tasas de ganancia. El $10 \%$ de los predios presenta alguna limitante. Los predios intestados requieren definir la certidumbre de la propiedad por medio de un juicio de sucesión (avalúo, designación de herederos y resolución). No obstante que el ayuntamiento promueve el registro de los predios privados con valor histórico (Imagen 3), mediante la deducción del impuesto predial sujeto a convenio. El largo proceso de investigación, catalogación e inscripción en el Registro Público de Monumentos y zonas Arqueológicos (Historia, 2013), desincentiva el interés y en consecuencia los propietarios abandonan los inmuebles

El universo de los baldíos expone que $74 \%$ de los predios privados no están en venta y ocupan una superficie de $145,748.38$ metros $^{2}$. Los predios en venta (3\%) agrupan una superficie de 10,221.58 metros ${ }^{2}$. Tienen barda perimetral, solo dos se utilizan como estacionamiento y se ubican en vialidades de acceso al centro histórico de la ciudad de Toluca: Paseo Tollocan; José María Morelos y Lerdo de Tejada. Los precios de los baldíos en venta, indican que, en el centro de la ciudad, el $\mathrm{m}^{2}$ oscila entre 15,000 y 20,000 pesos MN. En el borde exterior de Paseo Tollocan 6,000 pesos y Paseo Tollocan Norte 2,200.

Esta zona concentra mayor número de baldíos, el precio menor se explica por la pendiente que dificulta la urbanización y la dotación de servicios básicos. Significa que el equipamiento y los servicios condicionan las variaciones de los precios de los predios en venta. Aparentemente los propietarios carecen de interés para vender, el factor subyacente es la especulación. Este fenómeno artificial encarece y concentra bienes inmuebles de consumo final, incrementando el precio de los baldíos en el tiempo. La oferta de suelo depende del valor de los demás bienes, los impuestos y las expectativas de comercialización (Schiller, 1994). La determinación de la base gravable para calcular el monto de pago de los impuestos y derechos sobre los predios, compraventa, afectación o expropiación requiere avalúo catastral y comercial (México, Servicios 
Catastrales, 2015). Sin embargo, debido a las imperfecciones en el mercado de suelo urbano, el precio privado es significativamente mayor a los precios sociales (Jaramillo, 2003). El ejemplo emblemático de baldíos de propiedad privada, lo representa la antigua fábrica de jabón, por décadas se mantuvo sin uso, una vez vendido se encuentra en proceso de recuperación y no necesariamente con fines colectivos. Las limitantes para recuperar los baldíos privados en beneficio colectivo, identifican la especulación comercial y la carencia de fondos públicos. La reactivación de los predios no solo depende de la superficie, sino de los fondos económicos predestinados para proyectos de beneficio público.

\section{ESPACIO PÚBLICO Y BALDÍOS URBANOS}

El universo de intervención involucra a los tres niveles de gobierno, así lo define el plan nacional de desarrollo (GR-PND, 2013-2018) y la Nueva Agenda Urbana adoptada en la Conferencia Hábitat III (ONU-HABITAT III, 2016). En esta conferencia, México posicionó siete ejes, entre otros, combatir la desigualdad; nuevo marco legal para la gobernanza metropolitana; construcción de ciudades compactas; acción eficiente del suelo; promoción de una nueva cultura del espacio público y construcción de ciudades sostenibles para cuidar el entorno (GMX, 2016).

En este marco el Ayuntamiento de Toluca, planteó recuperar los espacios públicos para la convivencia y disfrute familiar (México, Plan de Desarrollo Urbano del Municipio de Toluca, 2003). No obstante que los bienes públicos pueden aprovecharse en términos de las disposiciones legales (Código Civil del Estado de México, 2012), las acciones se expresan en la remodelación de algunos edificios históricos y parques públicos. Aunque se cuenta con parques y zonas de reserva, destinadas a la recreación, contemplación y ocio público: Parque Vicente Guerrero; Alameda central Toluca; Alameda 2000; Parque Matlatzincas (El Calvario); Parque Metropolitano Bicentenario. Toluca y Parque Estatal Sierra Morelos, la superficie ocupada por zonas verdes o jardines que forman parte del espacio urbano, asciende a 74, 135 metros cuadrados. Es estratégico que las autoridades asimilen que la calidad del espacio público es un factor clave de la productividad urbana (García Vázquez, 2010), estructuran la ciudad, determinan la convivencia colectiva, proveen de un medio ambiente habitable (Saldarriaga Roa, 2002) e incrementan el valor de las propiedades.

\section{ESTRATEGIAS DE RECUPERACIÓN DE BALDÍOS URBANOS}

La recuperación de los predios baldíos depende del saneamiento jurídico de la propiedad inmobiliaria, lo cual permitiría poner en marcha proyectos específicos destinados al disfrute 
colectivo. Anticipando conflictos jurídicos, económicos y sociales, y en ausencia de políticas específicas se proponen estrategias normativas para recuperar los baldíos urbanos. Las primeras consisten en aplicar las disposiciones legales preexistentes en la norma mexicana; el segundo grupo de estrategias corresponde a disposiciones que deberán estar sujetas a un proceso legislativo:

\section{Estrategias con base en el marco normativo vigente:}

a) Proceso Penal: Inicia a través de la querella o la denuncia pública, prevista en el Código de Procedimientos Penales del Estado de México, el ofendido o toda aquella persona que tenga conocimiento de hechos que puedan ser constitutivos de una conducta delictiva, puede dar inicio la investigación (carpeta de investigación llamada anteriormente averiguación previa) sobre una presunta invasión del espacio público.

La ley la establece la condición de que la querella sea una violación directa que no afecte a terceras personas, únicamente procede en delitos no graves, es decir, el ayuntamiento como representante de la colectividad puede interponer querella ante la Agencia del Ministerio Público para recuperar los baldíos urbanos alegando el bien de la colectividad. En caso que la investigación se inicie por denuncia, cualquier ciudadano puede ejercerla, ya sea porque se trata de bienes de dominio público, o bienes de carácter privado, que representan la posible comisión de un delito, despojo y daño a la colectividad. El Ministerio Público deberá determinar si existen los elementos suficientes para solicitar la extinción de dominio (cesación de los derechos de propiedad a favor del dueño o poseedor), que permita reincorporar los bienes a la tenencia del Estado.

b) Proceso Civil: Este instrumento inscrito en el Código de Procedimientos Civiles del Estado de México, permite a cualquier persona física o moral, en este caso el Ayuntamiento de Toluca, interponer demanda de prescripción adquisitiva (usucapión) ante el abandono latente de los baldíos urbanos; para que sea procedente. El Ayuntamiento deberá tener posesión de los baldíos urbanos por un periodo superior a cinco años, de buena fe, de forma pacífica y con el carácter de propietario; aunado a lo anterior se deberá notificar al dueño o posesionario, quien tendrá derecho a ejercer su garantía de audiencia; una vez presentadas las pruebas la recuperación del baldío urbano queda en decisión de un órgano jurisdiccional.

c) Procesos No contenciosos: Además de las acciones administrativas y judiciales que adelanten los gobiernos municipales y estatal, es fundamental que establezcan y promuevan procesos de recuperación del espacio público, que faciliten a las comunidades acogerse a las normas por su propia voluntad. Brindar estas alternativas genera ejemplos para las comunidades y contribuye a la promoción de procesos de organización y participación comunitaria, a través de los 
cuales se identifican opciones de uso del espacio público que solucionan los conflictos, al tiempo que se mejora la convivencia. Estos representan los acuerdos entre gobierno y comunidad para la entrega voluntaria de espacios públicos cerrados ilegalmente por particulares.

Recuperar estas zonas, que normalmente pertenecen a barrios construidos legalmente para estratos medios y altos, implica un intenso y planeado trabajo de negociación con la comunidad de vecinos, en muchas ocasiones pueden sentir que la eliminación de las privadas los deja expuestos a robos, delincuencia y vandalismo.

d) Juicio de Amparo: Conforme a las reformas aplicadas a los artículos 103 y 105 de la Constitución Política de los Estados Unidos Mexicanos se otorga reconocimiento a los derechos difusos, también llamados derechos humanos de tercera generación, los cuales tiene por objeto proteger los bienes que atañen a la colectividad, es decir a sujetos pasivos que no conforman un sector poblacional identificable e individualizado. La pluralidad de los sujetos cuyo vínculo jurídico se ve lesionado o amenazado ante hechos genéricos, contingentes o mutantes que emanan de sujetos que deben una prestación genérica o indeterminada, como son los daños ambientales; en este caso el deterioro de la ciudad por el abandono de los baldíos urbano genera un daño indirecto y latente a la colectividad.

De conformidad al ordenamiento jurídico antes señalado, cualquier individuo, asociación, poder público, sindicato o comunidad pueden interponer el juicio de garantías ante el daño ocasionado. En caso de ser procedente el juicio de amparo (recurso jurídico similar al abeas corpus en el Derecho Anglosajón), ordenaría la re incorporación de los bienes al sector público.

e) Juicio de Expropiación: En conformidad a lo dispuesto en la Ley de Expropiación del Estado de México, cuando un bien es requerido por causa de utilidad pública para: I. La apertura, ampliación, prolongación, alineamiento o mejoramiento de calles, calzadas, puentes, túneles, carreteras y vías que faciliten el tránsito de personas o vehículos; Il. Embellecimiento, ampliación y saneamiento de las poblaciones; la construcción, ampliación, prolongación o mejoramiento de plazas, parques, jardines, mercados, instalaciones deportivas, hospitales, oficinas públicas, escuelas, rastros, cementerios, áreas para estaciones de seguridad pública y para reserva ecológica y cualquier obra destinada a prestar servicios públicos; establecimiento, funcionamiento o mantenimiento de éstos, así como la administración por el Estado o municipios de uno existente que beneficie a la colectividad para evitar su abandono o suspensión; podrá dar inicio a un juicio de expropiación, a través del cual, el propietario será privado de sus bienes previa indemnización legal. Aunque este procedimiento se encuentra previsto en la legislación Estatal, en pocas ocasiones es aplicado, dado que los proyectos que justifican la utilidad pública suelen no reunir los requisitos legales. 


\section{Estrategias con base en la Nueva Ley de Ordenamiento Territorial y Urbano:}

a) Creación de un cuerpo normativo de carácter estatal, (Código de Regulación de Valores Inmobiliarios del Estado de México) que confiera a la legislatura local la facultad de regular los precios del mercado de bienes inmuebles, teniendo competencia superior a cualquier bando municipal, que tenga por objeto unificar los valores catastrales y los valores comerciales de los bienes urbanos, evitando la especulación comercial.

b) Constitución del Consejo de Valoración de Bienes Urbanos, cuya función será transparentar los resultados de los mercados inmobiliarios en el Estado de México, teniendo como principales atribuciones las siguientes:

i. Elaboración del Índice de Precios de Compraventa; ii. Publicación de los informes del mercado inmobiliario; iii. Emitir avalúos de bienes urbanos; iv. Coadyuvar con los Catastros Municipales y Secretarías de Desarrollo Municipal en la elaboración del inventario e índice de baldíos urbanos; v. Participar proactivamente en la actualización de los padrones catastrales municipales; vi. Emisión de los certificados de Potencial Adicional Constructivo (tienen por objeto que los propietarios de baldíos urbanos que no le den uso efectivo, paguen un sobre precio dado por la tasa de incremento del valor del inmueble).

c) Institucionalizar la aplicación de la política de recuperación de plusvalías, entendida como la totalidad de los incrementos del valor de la tierra atribuibles a los esfuerzos de la comunidad para convertirlos en recaudación pública por la vía fiscal en beneficio de los ocupantes o de la comunidad (Smolka \& Furtado, Recuperación de Plusvalías en América Latina. Alternativas para el desarrollo urbano., 2001), este instrumento legal favorecerá la liberación de espacios y activos urbanos que no tienen un valor de uso, la recaudación, el mejoramiento de la imagen de la ciudad y la gestión de espacios aprovechables según sus características, para ello se deberán aplicar los mecanismos siguientes:

i. Aplicación efectiva del impuesto por Contribución de Mejoras (permite internalizar los costos de equipamiento y obras a los titulares de los inmuebles que se ven beneficiados con un aumento del valor de su inmueble); ii. La concentración de inversiones públicas en infraestructuras en las áreas de renta más elevadas que permita la captación de un impuesto mayor de las personas que se ven beneficiadas por las mismas y a la vez recuperar las áreas urbanizadas sin ocupación.

d) La creación del Consejo Ciudadano para la Ocupación y Fomento de Espacios Urbanos, que tendrá por objeto impulsar la participación ciudadana, de forma que sus opiniones sean recabadas y plasmadas en proyectos tendientes a satisfacer las necesidades colectivas de acuerdo a su edad y los 
espacios susceptibles a ser recuperados, que generen un impacto en el diseño paisajístico, equipamiento, iluminación, forestación y rejuvenecimiento de la ciudad.

e) Reforma a la Ley de Extinción de Dominio del Estado de México, que otorgue facultades al municipio a través del Síndico Procurador para interponer formal demanda en contra del titular de los derechos reales (derechos de propiedad sobre bienes inmuebles), por mantenerlos desocupados por un periodo superior a diez años, lo anterior reducirá la especulación comercial evitando el incremento de los predios baldíos sin valor de uso cierto.

f) Establecimiento del sistema de usufructo, a través del cual el propietarios de los bienes urbanos transmite la posesión del inmueble al Estado, quien administra los bienes baldíos, otorgándoles una función social, a cambio el usufructuario se encuentra exento del pago de cualquier contribución generada a lo largo del convenio, teniendo el ayuntamiento la preferencia en la compra del inmueble.

\section{DISCUSIÓN DE RESULTADOS}

Los resultados de la presente investigación, muestran que la superficie total de los predios baldíos corresponde a 160, 363.85 metros $^{2}$ (16.07 ha). La superficie ocupada por los predios privados que no están en venta $\left(145,748.38\right.$ metros $\left.^{2}\right)$, representa más de $90 \%$ de la superficie total ocupada por 180 baldíos. La vida útil de estos inmuebles es extensa y en espera de ser consumidos. El suelo se posiciona como forma de inversión que incide en la valoración inmobiliaria; (Godoy, 2006) y en la variabilidad de la renta urbana (Barbieri, Fina, \& Subirats, 2011). Los baldíos urbanos están a la espera de la oferta inmobiliaria y en general del mejor postor. El número y la superficie de los predios baldíos que no están a la venta, inscribe los impactos negativos en los ámbitos de la salud y la economía, lo cual demanda eliminar los focos de insalubridad y la especulación comercial. La recuperación de los predios baldíos con fines de beneficio colectivo, no puede realizarse de manera unilateral, el derecho a la propiedad privada se encuentra regulado en el artículo veintisiete de la Constitución Política de los Estados Unidos Mexicanos, y a su vez es en la Declaración Universal de los Derechos Humanos. Este derecho mediado por la variación de las rentas en el mercado del suelo, determina que los baldíos urbanos se conviertan en objeto de especulación comercial.

Si la recuperación de los predios baldíos se basa en el mercado de productos, entonces la tierra es un producto final, la oferta de este producto es función de los demás bienes, los impuestos y las expectativas de comercialización (Schiller, 1994). Lo anterior explica que los gobiernos muestren poco interés por impulsar áreas urbanas deterioradas; puesto que implementar políticas de recuperación y ordenamiento requiere fuertes inversiones para revertir la obsolescencia de edificios 
abandonados, nuevos equipamientos, mejoramiento y ampliación de la infraestructuras, entre otros (Godoy, 2006). No existe receta aplicable a la tierra vacante, en la mayoría de los casos, la mejor estrategia es mantener los terrenos desocupados; en otros, las condiciones exigen medidas que prevengan o alivien problemas ambientales y de salud pública. Los baldíos urbanos deben ser objeto de una cuidadosa planificación, representan una oportunidad para la revitalización de la ciudad (Article, 1999). Los desafíos deben solventar las contradicciones legales y normativas, así como estrategias innovadoras que fortalezcan las directrices de la densificación urbana y las finanzas públicas.

\section{CONCLUSIONES}

No obstante que las prioridades acotan regularizar la certidumbre jurídica de la propiedad e incrementar las contribuciones por este concepto. En la entidad y en el municipio de Toluca se carece de un ordenamiento que permita recuperar los predios baldíos sin valor de uso cierto, lo cual agudiza su abandono por largos períodos de tiempo y la especulación comercial. El marco de la Nueva Ley de ordenamiento territorial y desarrollo urbano, demanda diseñar y aplicar políticas de nueva generación, sostenidas en estrategias innovadoras que coadyuven en los procesos de densificación urbana a mediano y largo plazo.

Los gobiernos municipales tienen la responsabilidad de controlar y gestionar los espacios para beneficio colectivo, mediante la reinserción de suelo útil, los beneficios repercutirán no solo en la densificación, sino también en la habitabilidad urbana y en las oportunidades de inversión.

Los gobiernos deberán priorizar zonas que tengan alto impacto económico y complementen iniciativas de proyectos de recuperación del espacio público. El derecho a la propiedad privada proscribe la recuperación de predios baldíos, en estas condiciones la política pública y las acciones extra judiciales, dotan de facultades para incorporar los predios urbanos baldíos a la dinámica urbana.

La reinserción de los baldíos urbanos no se limita a la recuperación de plusvalías, es imperante la gestión de espacios públicos, atender el déficit de jardines urbanos, remozar edificaciones patrimoniales asignándoles funciones en razón al tamaño, diseño y requerimientos sociales. Se requiere seleccionar los predios que por sus dimensiones y situación administrativa, permitan desarrollar programas y proyectos en beneficio colectivo. Explorar a través de la consulta ciudadana necesidades y propuestas, para que la recuperación de predios baldíos y los espacios públicos cumplan con su finalidad. 
Este estudio contribuye con el análisis de un tema escasamente trabajado, la identificación de los baldíos urbanos en la ciudad de Toluca fue complicada, debido a la carencia de información, sin embargo el procedimiento utilizado sienta bases para mejorarse y replicarse en otros municipios. La identificación de los vacíos legislativos en materia de baldíos urbanos, la especulación comercial, la enajenación fraudulenta y la sub utilización que opera al amparo de las debilidades de la legislación vigente. Define la naturaleza normativa de las estrategias de recuperación que se proponen. Sin embargo, la implementación está sujeta a un proceso de discusión, acuerdo y aprobación en los distintos ámbitos de gobierno del Estado de México.

\section{BIBLIOGRAFÍA}

ARTICLE, L. L. (1999). Recuperado el 28 de 05 de 2015, de http://www.lincolninst.edu/pubs/350 ElInstituto-Lincoln-celebra-su-aniversario-de-plata-en-medio-de-conferencias-internacionales

BARBIERI, N., FINA, X., \& SUBIRATS, J.. Culture and Urban Policies: Dynamics and Effects of Cultural Third Sector Interventions in Barcelona. Métropoles, 2-18. (2011)

CASTILLO, F.. Yucatán en corto. Obtenido de http://florcastillo.mx/noticias/proponen-elevarsanciones-a-propietarios-de-terrenos-baldios.html(28 de 05 de 2015)

CONTRERAS Gatica, Y.. La recuperación urbana y residencial del centro de Santiago: Nuevos habitantes, cambios socioespaciales significativos. EURE, 89-113. (2011)

CUBILLOS, L.. Interculturalidad y Políticas Públicas en Educación: Reflexiones desde Santiago de Chile. Santiago: Estudios Pedagógicos Valdivia. (2006)

DEMATTEIS, G.. Sul crocevia della terriorialitá urbana (Milano). (1999)

EARTH, G.. Google Earth. Obtenido de http://earth.google.es/(25 de 07 de 2015)

FAUSTO, A., \& RÁBAGO, J.. habitat. Recuperado el 28 de 05 de 2015, de http://habitat.aq.upm.es/boletin/n21/aafau.html. (2001)

FERNÁNDEZ, R. Gestión Ambiental de Ciudades. Teoría Crítica y aportes metodológicos. México: Red de Formación Ambiental para América Latina y el Caribe. (2006).

GODOY, S. M. (0. J. Investigación, Ed.) Recuperado el 2015, de http://arq.unne.edu.ar/publicaciones/comunicaciones06/ponencias/godoy.pdf. (2006).

HISTORIA, I. N. Instituto Nacional de Antropología e Historia. Recuperado el 20 de 05 de 2015, de http://inah.gob.mex/boletines/249-monumentos-historicos/6152-incorporan-10-mil-edificioshistoricos-al-registro-publico. (2013) 
HURTADO Muñoz, V. Análisis de la renovación urbanacomo estrategia de recuperación del centro histórico de Bogotá. Estudio de caso Barrio Santa Bárbara Colonial (Nueva Santa Fe), en el periodo 1976-2000. (F. d. Gobierno, Ed.) 76. (2011).

INSTITUTO NACIONAL DE GEOGRAFÍA ESTADÍSTICA E INFO. Resulatados definitivos del Censo de Población 2010. Estado de México: INEGI. (2010).

JARAMILLO, S. Los fundamentos económicos de la participación en plusvalías. Los Andes: CIDE. (2003).

MÉXICO, G. d. (S. d. Metropolitano, Ed.) Recuperado el 05 de 27 de 2015, de http://portal2.edomex.gob.mx/sedur/planes_de_desarrollo/planes_municipales/toluca/index.htm. (2003).

MÉXICO, G. d. Servicios Catastrales, htm. Recuperado el 10 de 06 de 2015, de Instituto de Información e Investigación Geográfica, Estadística y Catastral, Secretaría de Finanzas: http://portal2.edomex.gob.mx/igecem/productos y servicios/servicios catastrales/servicios/index.htm. (2015).

OROZCO Hernández, M. E., \& Tapia Quevedo, J. (s.f.). Reserva Territorial y construcción social del espacio Urbano. Zona metropolitana de Toluca, Estado de México. En Orozco, \& Sánchez (Edits.). Toluca: Universodad Autónoma del Estado de México. (2015).

SÁNCHEZ Muñoz, H. E. Gestión de áreas verdes mediante la recuperación de baldíos, caso: Ciudad de Toluca. Toluca. (2011).

SCHILLER, B. R. Principios Esenciales de Economía. Madrid: McGrawHill. (1994).

SMOLKA, M., \& Amborski, D. Recuperación de plusvalías para el desarrollo urbano: una comparación inter- americana. Eure, $X X I X(88)$, 55-77. (2003).

SMOLKA, M., \& Furtado, F. Recuperación de Plusvalías en América Latina. Alternativas para el desarrollo urbano. Santiago, Chile: eurelibros. (2001).

SONORA, I. C. (C. N. Hacendaria, Ed.) Obtenido de http://www.indetec.gob.mx/cnh/propuestas/25.htm. (27 de 05 de 2015).

WIEL, M. (2012). Maitriser la rente immobiliere. Piloter la rente immobiliere, clef de la politique urbaine. Futuribles(391), 47-59. Recuperado el 27 de 05 de 2015, de http://dialnet.uniroja.es/servlet/autor?codigo=3203193

Estados Unidos Mexicanos.- Presidencia de la República. LEY GENERAL DE ASENTAMIENTOS HUMANOS, ORDENAMIENTO TERRITORIAL Y DESARROLLO URBANO. Nueva Ley publicada en el DOF el 28 de noviembre de 2016.

Instituto Nacional de Estadística, Geografía e Informática (INEGI). Características del entorno urbano 2014. Fecha de elaboración: 08/12/2015.

http://www.beta.inegi.org.mx/proyectos/encotras/cleu/2014/

GOBIERNO DE LA REPÚBLICA, Plan Nacional de Desarrollo 2013-2018. Estado Unidos Mexicanos

GOBIERNO DE MÉXICO.2016. La Nueva Agenda Urbana. Derecho a la Ciudad, Accesibilidad Universal y Enfoque de Género, aportaciones de México a la Nueva Agenda Urbana. Boletín, publicado el 24 
de octubre de 2016. http://www.gob.mx/nuevaagendaurbana/articulos/derecho-a-la-ciudadaccesibilidad-universal-y-enfoque-de-genero-aportaciones-de-mexico-a-la-nueva-agenda-urbana75856

ONU-HABITAT III. Adopted Draft of the New Urban Agenda, 20 October 2016. Quito, Ecuador, https://habitat3.org/the-new-urban-agenda

Trabalho enviado em 30 de janeiro de 2018

Aceito em 28 de outubro de 2021 